\title{
Differences in influenza virus receptors in chickens and ducks: Implications for interspecies transmission
}

\author{
Suresh V Kuchipudi ${ }^{1,2}$, Rahul Nelli ${ }^{1}$, Gavin A White ${ }^{1}$, Maureen Bain ${ }^{2}$, Kin Chow Chang ${ }^{1}$ and \\ Stephen Dunham ${ }^{1 *}$ \\ ${ }^{1}$ School of Veterinary Medicine and Science, University of Nottingham, Sutton Bonington Campus, College Road, \\ Loughborough, Leicestershire LE12 5RD, UK, ${ }^{2}$ Institute of Comparative Medicine, Faculty of Veterinary Medicine, \\ University of Glasgow, Bearsden, Glasgow, G61 1QH, UK \\ *Correspondance to: Stephen Dunham, Email: stephen.dunham@nottingham.ac.uk, Tel: +44 115 9516464, Fax: +44 \\ 1159516580
}

Received 09 September 2008, Revised 13 December 2008, Accepted 22 December 2008, Published online 16 January 2009

J Mol Genet Med (2009), 3(1), 143-151

(C) Copyright The Authors: This is an open access article, published under the terms of the Creative Commons Attribution Non-Commercial License (http://creativecommons.org/licenses/by-nc/2.0/uk/). This license permits noncommercial use, distribution and reproduction of the article, provided the original work is appropriately acknowledged with correct citation details.

\begin{abstract}
Avian influenza viruses are considered to be key contributors to the emergence of human influenza pandemics. A major determinant of infection is the presence of virus receptors on susceptible cells to which the viral haemagglutinin is able to bind. Avian viruses preferentially bind to sialic acid $\alpha 2,3$-galactose (SA 22,3-Gal) linked receptors, whereas human strains bind to sialic acid $\alpha 2,6$-galactose (SA $\alpha 2,6-$ Gal) linked receptors. While ducks are the major reservoir for influenza viruses, they are typically resistant to the effects of viral infection, in contrast to the frequently severe disease observed in chickens. In order to understand whether differences in receptors might contribute to this observation, we studied the distribution of influenza receptors in organs of ducks and chickens using lectin histochemistry with linkage specific lectins and receptor binding assays with swine and avian influenza viruses. Although the intestinal epithelial cells of both species expressed only SA $\alpha 2,3-G a l$ receptors, we found widespread presence of both SA $\alpha 2,6-G a l$ and SA $\alpha 2,3-G a l$ receptors in many organs of both chickens and ducks. Co-expression of both receptors may allow infection of cells with both avian and human viruses and so present a route to genetic reassortment. There was a marked difference in the primary receptor type in the trachea of chickens and ducks. In chicken trachea, SA 2,6-Gal was the dominant receptor type whereas in ducks SA 2 2,3-Gal receptors were most abundant. This suggests that chickens could be more important as an intermediate host for the generation of influenza viruses with increased ability to bind to SA $2,6-\mathrm{Gal}$ receptors and thus greater potential for infection of humans. Chicken tracheal and intestinal epithelial cells also expressed a broader range of SA $\alpha 2,3-$ Gal receptors (both $\beta(1-4)$ GlcNAc and $\beta(1-3)$ GalNAc subtypes) in contrast to ducks, which suggests that they may be able to support infection with a broader range of avian influenza viruses.
\end{abstract}

KEYWORDS: Host receptors, influenza, interspecies transmission, chicken, duck

\section{INTRODUCTION}

Influenza $A$ is one of the most important infectious diseases of humans and is responsible for recurrent seasonal disease epidemics. Wild birds are the natural reservoir for all recognized subtypes of influenza A and as such present a potential route for the emergence of new viral strains which can cause human disease. If such newly 
emerged viruses acquire the ability to transfer effectively from human to human they may lead to pandemic outbreaks with widespread illness and mortality. The H1N1 influenza pandemic of 1918 caused 20 to 50 million deaths on a global scale, making it the single most devastating disease outbreak in human history (Johnson and Muller, 2002). This pandemic is believed to have been caused by an avian virus that crossed the species barrier to infect humans and underwent subsequent adaptation to a new host (Taubenberger et al, 2005). The resultant virus acquired exceptional virulence with the ability to replicate in the absence of trypsin, induce death in mice and grow rapidly in human epithelial cells (Tumpey et al, 2005). Novel influenza viruses may also arise due to concurrent infection with different virus strains through reassortment of viral RNA segments (Horimoto and Kawawoka, 2005). Reassortment of human and avian influenza A viruses is thought to have generated the pandemic viruses of 1957 and 1968 (Webster et al, 1997). Avian influenza viruses are therefore likely to continue to play a significant role in the emergence of new human influenza strains (Perdue and Swayne, 2005). More recently, human infections have been caused by the emergence of avian H5N1 viruses, initially in Hong Kong in 1997 (Claas et al, 1998; Subbarao et al, 1998). Subsequently, H5N1 has reemerged as a significant threat to human health, with over 385 confirmed cases and 243 deaths (WHO data, June 2008). These cases have demonstrated that avian viruses can directly infect humans without the need of intermediate hosts such as pigs. However, the precise molecular basis for the efficient transmissibility of avian influenza viruses to mammals is not fully understood.

A major determinant of the ability of influenza viruses to infect cells is the expression of the appropriate host cell receptor to which viral haemagglutinin can bind. Consequently, a crucial hurdle that influenza A viruses need to overcome when crossing a species barrier is the acquisition of the ability to utilize alternate host cell receptors (Ito, 2000). Influenza virus receptors on host cells are glycosylated oligosaccharides that terminate in sialic acid (SA) residues which are bound to glycans through an $\alpha 2,3$ or $\alpha 2,6$ linkage, mediated by sialyltransferases that are expressed in a cell- and speciesspecific manner (Gagneux et al, 2003). Avian influenza viruses have been shown to preferentially bind to SA receptors that are linked to galactose by an $\alpha 2,3$ linkage (SA 2,3-Gal), while human and classical swine viruses show preference for receptors with an $\alpha 2,6$ linkage (SA 22,6-Gal) (Gagneux et al, 2003; Matrosovich et al, 2004). Among avian influenza viruses, chicken and duck viruses have been shown to further differ in their ability to recognize the structure of the third sugar moiety in SA 2,3-Gal terminated receptors. A collection of avian influenza viruses from chickens and ducks has been studied for their binding affinities to synthetic receptor analogues in vitro. A marked contrast in preferential binding was noted, where influenza viruses from chickens preferentially bound to synthetic sialylglycopolymer containing Neu5Ac- $\alpha(2-3)$ Gal- $\beta(1-4)$ GlcNAc, whereas viruses from ducks displayed a higher affinity for Neu5Ac- $\alpha(2-3)$ Gal- $\beta(1-3)$ GalNAc containing polymer (Gambaryan et al, 2003).
Ducks and chickens are the major aquatic and terrestrial hosts for a wide variety of influenza viruses and are valuable natural models to study influenza, with ducks displaying resistance to disease, but chickens, in contrast, showing relatively high susceptibility. Receptors are important determinants of virus entry and differences in receptor distribution between hosts could therefore account for variation in susceptibility to infection, including the contrasting outcomes following infection with many strains of highly pathogenic avian influenza (HPAI). Influenza virus receptors in chickens and ducks have previously been studied by virus binding assays using extracted total gangliosides from plasma membranes of respiratory and intestinal epithelial cells (Gambaryan et al, 2002). Based on the binding of human influenza virus with SA 2 ,6-Gal receptor specificity, it was found that chicken epithelial cells, but not duck epithelial cells, express SA 2,6-Gal receptors. However, the receptor distribution across intact mucosal surfaces and organ systems was not studied. More recently, chicken trachea and duodenum have been studied using lectin binding assays followed by conventional immunohistochemistry and light microscopy (Wan and Perez, 2006). Despite this, detailed information on the distribution of influenza virus receptors in these important avian hosts is lacking. To further evaluate the potential role of receptor distribution in ducks and chickens in determining susceptibility to infection, and the potential of these species to support infection of viruses

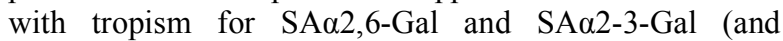
therefore act as "mixing vessels") we have examined in detail the anatomical distribution of influenza A receptors in key organs of both species.

\section{MATERIALS AND METHODS}

\section{Animal tissues}

Animals for this study comprised four 35-40 wk old, commercial layer chickens (Glenrath Farms Ltd, East Lothian, UK), four 4 wk old broilers (PD Hook Hatcheries, Bampton, Oxfordshire, UK), two 3 wk old and four 6 wk old Pekin ducks (Cherry Valley Farms, Rothwell, Lincolnshire, UK). The animals were euthanased and samples from trachea, lungs, heart, kidney, brain, skeletal muscle, small and large intestine collected into buffered neutral formalin.

\section{Lectin histochemistry}

Tissue samples were dehydrated and cleared using a histokinette (Leica TP 1020) before being embedded in paraffin wax. After embedding, the tissues were sectioned using a rotary microtome (Leica RM 2255) with a specimen feed of $5 \mu \mathrm{m}$. Lectin histochemistry using linkage specific lectins was carried out with minor modifications of a method described previously (Shinya et al, 2006). Lectins used in the study were: Sambucus nigra agglutinin (SNA) specific for SAa2,6-Gal (Shibuya et al, 1987), Maackia amurensis I (MAA I) and Maackia amurensis agglutinins (MAA II) which are specific for SA $\alpha 2,3-G a l \beta(1-4) G l c N A c$ and SA $\alpha 2,3-G a l \beta(1-3) G a l N A c$ respectively (Konami et al, 1994) (all provided by Vector Laboratories, Burlingame, CA). Sections were pre-soaked in TBS and blocked using a biotin-streptavidin blocking kit (Vector Laboratories) according to manufacturer's 
instructions, followed by $4^{\circ} \mathrm{C}$ overnight incubation with fluorescein isothiocyanate (FITC) labelled SNA or FITC labelled MAA I, and biotinylated MAA II lectin each at a concentration of $10 \mu \mathrm{g} / \mathrm{ml}$. After three washes with TBS, the sections were incubated with streptavidin-AlexaFluor594 conjugate (Molecular Probes Inc, Eugene, OR) for $2 \mathrm{hrs}$ at room temperature (RT). The sections were washed and then mounted with ProLong Gold antifade reagent with 4', 6-diamino-2- phenylindole, dihydrochloride (DAPI; Molecular Probes Inc, Eugene, OR). Negative controls were performed omitting the primary reagents. To rule out nonspecific binding of the lectins, tissue sections were treated, prior to lectin staining, with Sialidase A (N-acetylneuraminate glycohydrolase; Prozyme, San Leandro, CA), which cleaves all non-reducing terminal sialic acid residues in the order $\alpha(2,6)>\alpha(2,3)>$ $\alpha(2,8)>\alpha(2,9)$. The sections were imaged using confocal microscopy (Leica TCS SP2 AOBS). Differences in receptor distribution on the mucosal lining of tracheae or intestines were quantified using LCS Lite software. Mean energy values were measured for each fluorochrome in a representative area of the mucosa and corrected by subtracting background energy values.

\section{Receptor binding assays}

Receptor binding assays with $\mathrm{H} 1 \mathrm{~N} 1$ classical swine strain (A/Sw/Iowa/15/30), a subtype closely related to the human 1918 pandemic influenza virus (Tumpey et al, 2004), and a H2N3 low pathogenic avian strain (A/mallard duck/England/7277/06) were performed by a previously reported method, with minor modifications (Couceiro et al, 1993). Briefly, paraffin embedded $5 \mu \mathrm{m}$ sections of chicken and duck tracheae, small and large intestines were deparaffinised in xylene and rehydrated by alcohol. Sections were incubated with avian or swine influenza virus for two hours at $37^{\circ} \mathrm{C}$. The sections were washed, blocked with goat serum, and incubated with mouse monoclonal antibody to influenza nucleoprotein (Abcam, Cambridge, UK) at 1:1000 dilution for one hour at RT followed by FITC-labelled goat anti-mouse IgG (Abcam, Cambridge, UK) at 1:500 dilution for two hours at RT. After three further washes with TBS, the sections were mounted with ProLong Gold antifade reagent with DAPI and scanned by confocal microscopy (Leica TCS SP2 AOBS). Negative controls were performed by omitting the initial incubation with virus or primary antibody.

\section{RESULTS AND DISCUSSION}

We conducted an extensive examination of influenza virus receptor distribution in a range of tissues from chickens and ducks. No difference in the reported results was observed due to the age or source of animals, and the receptor distribution was consistent between individual animals within each species. Using lectin staining, we found widespread presence of both SA 2 2,6-Gal (SNA) and SA $2,3-\mathrm{Gal}$ (MAA II) receptors in a range of tissues from each species, suggesting that these organs may be potential targets for both avian and human influenza viruses (Figure 1). The expression of SA 2,6-Gal receptors in duck tissues is in contrast to a previous study using virus binding assays which reported that plasma membranes isolated from duck respiratory and intestinal epithelial cells did not express SA 2 2,6-Gal-terminated receptors (Gambaryan et al, 2002). The exact reason for this difference is not clear, however the previous study used mallard ducks rather than Pekin ducks used in this study, and the results were based on virus, rather than lectin binding, to isolated cell membranes rather than intact cells. Thus it is possible that a difference in the methodology or choice of duck breed may be responsible.

Although there is widespread distribution of both receptor types in both chickens and ducks, there are clear differences in their spatial distribution within organs between the two species. In the vascular endothelium of the kidney, both SA $\alpha 2,6-G a l$ and SA $\alpha 2,3-G a l$ receptors were found in duck cells, but only the SAa2,6-Gal receptor type was found in the corresponding region in chicken kidney. The significance of this difference is not clear, however presence of SA $22,6-\mathrm{Gal}$ receptors in the vascular endothelium in both species indicates that these cells can be potentially infected by mammalian-like influenza viruses and possibly play a role in the haematogenous spread of the virus. Tubular cells of duck kidney expressed both SA $\alpha 2,6-\mathrm{Gal}$ and SA $\alpha 2,3-\mathrm{Gal}$ receptors, whereas chicken kidney tubular cells expressed either SA 2,6-Gal or SA $\alpha 2,3-G a l$ receptors, but no coexpression was observed. The endocardium, meninges and muscle fibres of both species co-expressed both subtypes of receptor (Figure 1). The expression of both host receptors in such tissues in chickens and ducks suggests that these avian hosts could possibly serve as "mixing vessels" for virus reassortment following co-infection by human and avian viruses.

Chicken and duck intestine predominantly expressed the SA 2 2,3-Gal receptor type across the epithelial lining of villi (Figure 2A), as previously reported (Ito and Kawaoka, $2000 \&$ Wan and Perez, 2006). The measurement of fluorescent mean energy values along the epithelial lining of the mucosa showed no significant presence of SA $\alpha 2,6-$ Gal in intestines of either chicken or duck. This finding reaffirms the avian digestive tract as a major predilection site for avian influenza virus replication. The receptor distribution in the large intestine of both avian species was very similar to the small intestine (data not shown).

The major species difference that we observed between chicken and duck in the relative distribution of SAa2,3Gal and SA 2,6-Gal receptors was along the tracheal epithelium. In chicken tracheal epithelium, SA $\alpha 2,6-\mathrm{Gal}$ (with SNA binding) was the dominant receptor type whereas in ducks the SA 2 2,3-Gal receptor (with MAA II binding) was more abundant in the ciliated cells of the tracheal epithelium (Figure 2B). Based on mean fluorescent energy values, it was found that the ratio of SA 2 2,6-Gal to SA $2,3-\mathrm{Gal}$ in chicken trachea was approximately 10:1 whereas in duck the ratio was 1:20. The tracheal mucous glands of both chicken and duck predominantly expressed SA $\alpha 2,6-G a l$ receptor type. The observed difference in dominant receptor type between chickens and ducks was confined to the upper airway (trachea). In the bronchi and bronchioles the clear presence of both receptor types was found in both species. Chicken alveolar cells expressed both receptor types; the 

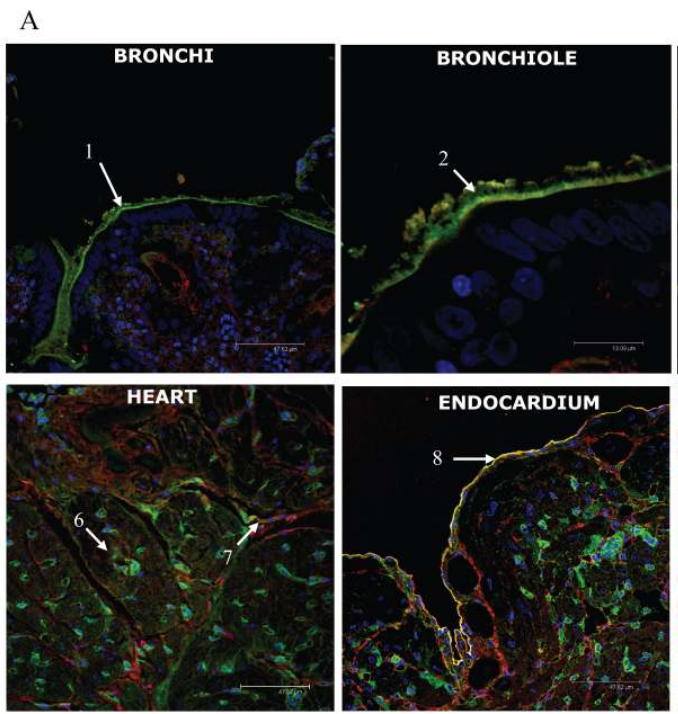

B
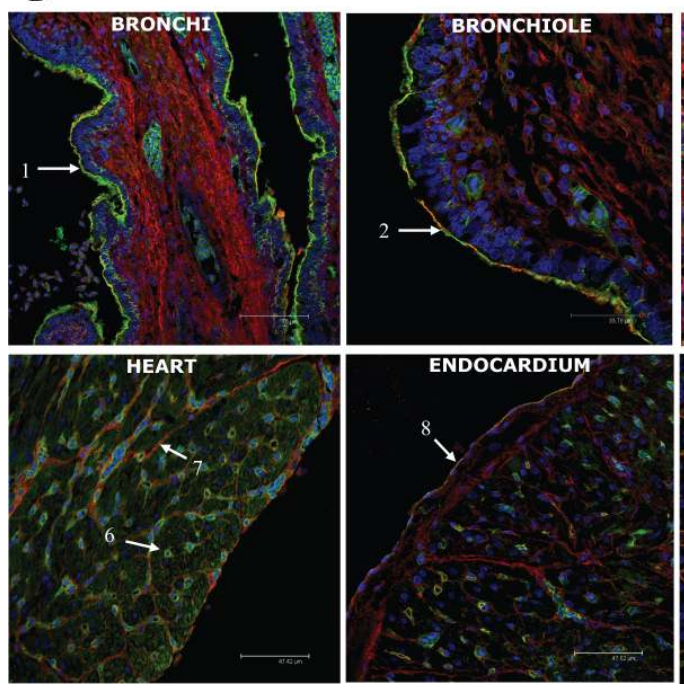
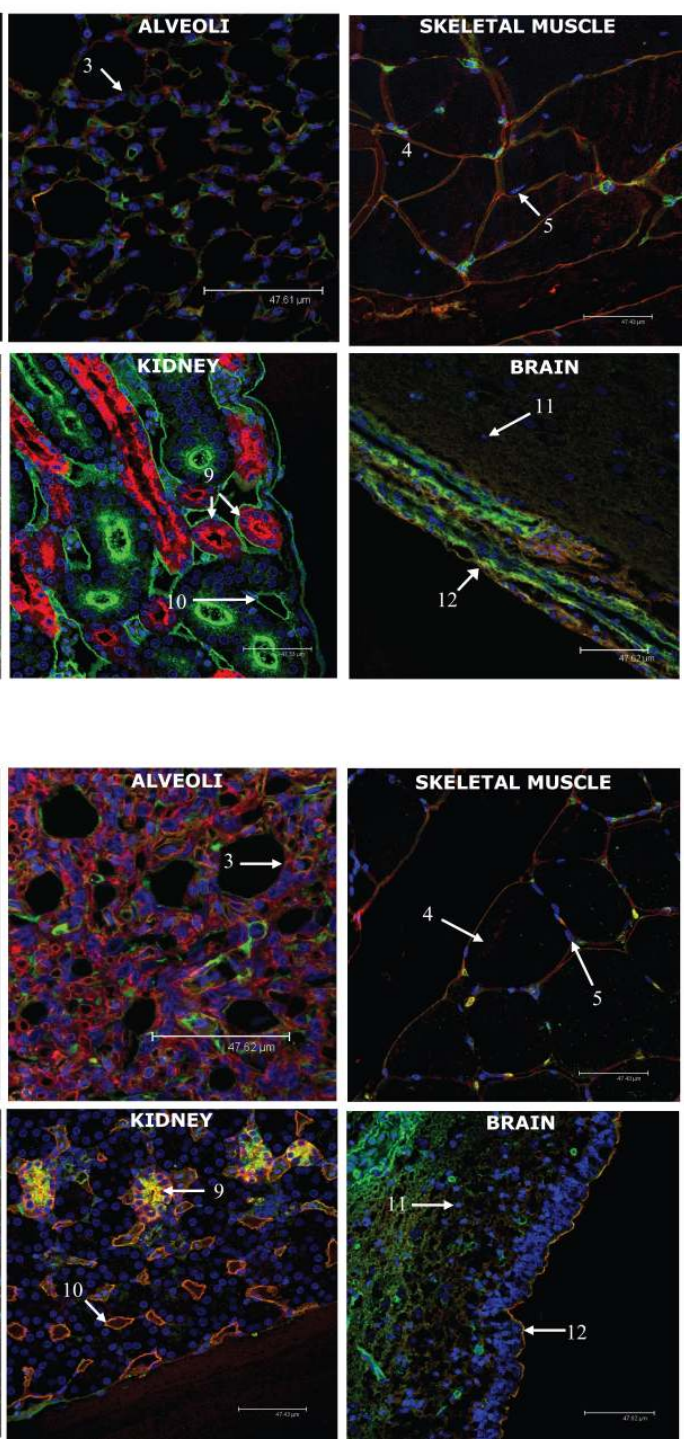

Figure 1. Widespread presence of SA $\alpha 2,6 \mathrm{Gal}$ (SNA lectin) and SA $\alpha 2,3 \mathrm{Gal}$ (MAA II lectin) receptors in many of organs of chickens (A) and ducks (B). Composite confocal images show distribution of SA $\alpha 2,6 \mathrm{Gal}$ receptors (green) and SA $\alpha 2,3 \mathrm{Gal}$ receptors (red) with nuclear staining (blue). 1. bronchial epithelium, 2. bronchiolar epithelium, 3. alveolar lining, 4. muscle fibre, 5. nucleus, 6. myocardium, 7. connective tissue, 8. endocardium, 9. convoluted tubule 10. vascular endothelium,, 11. neuronal tissue, 12. meninges.

precise staining pattern was more difficult to determine in duck alveoli due to the extensive presence of vascular tissue which tended to obscure the alveolar staining, however, both receptors could be seen clearly in some areas of the alveoli (Figure 1). The dominant SA $\alpha 2,6-\mathrm{Gal}$ receptor expression pattern in chicken trachea was in contrast to a previous study (Wan and Perez, 2006) which, using lectin binding, found that $85 \%$ of the epithelial cells in chicken trachea were positive for SA $\alpha 2,3-$-Gal receptors, while only $10 \%$ were positive for SA $2,6-6 a l$ receptors. However, it is in agreement with the findings of Gambaryan et al (2002), who reported that human influenza viruses with SA 2,6-Gal specificity bound to cell membranes isolated from chicken (but not duck) tracheal cell membranes. A possible explanation for the discrepancy in the reported receptor distribution in chicken trachea could be the source of the lectin used. Lectins from different suppliers may show different binding specificities; in particular the source of MAA has been shown to significantly affect specificity (Nicholls et al, 2007). Glycan microarray screening of lectins from the supplier used for our study confirms that these lectins bind with a high degree of specificity to the appropriate sialic acid linkages (http://www.functionalglycomics.org). It is also possible that this study was able to detect lower levels of receptor expression due to the methodology used (confocal microscopy with fluorescent detection compared with immunohistochemistry). We found that the observed predominance of SAa2,6-Gal in chicken trachea was consistent in different ages and breed of chicken. We also 
A
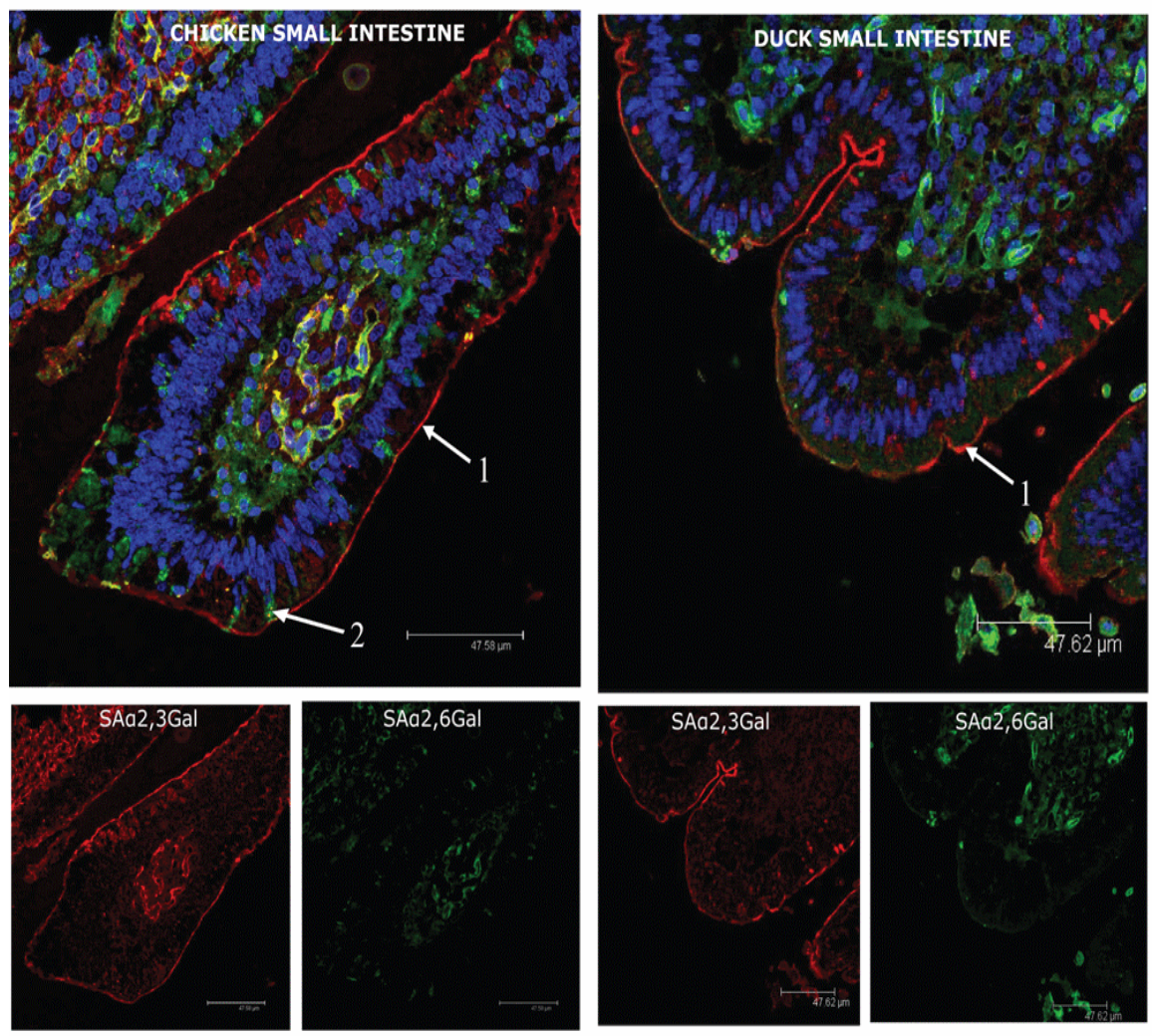

B
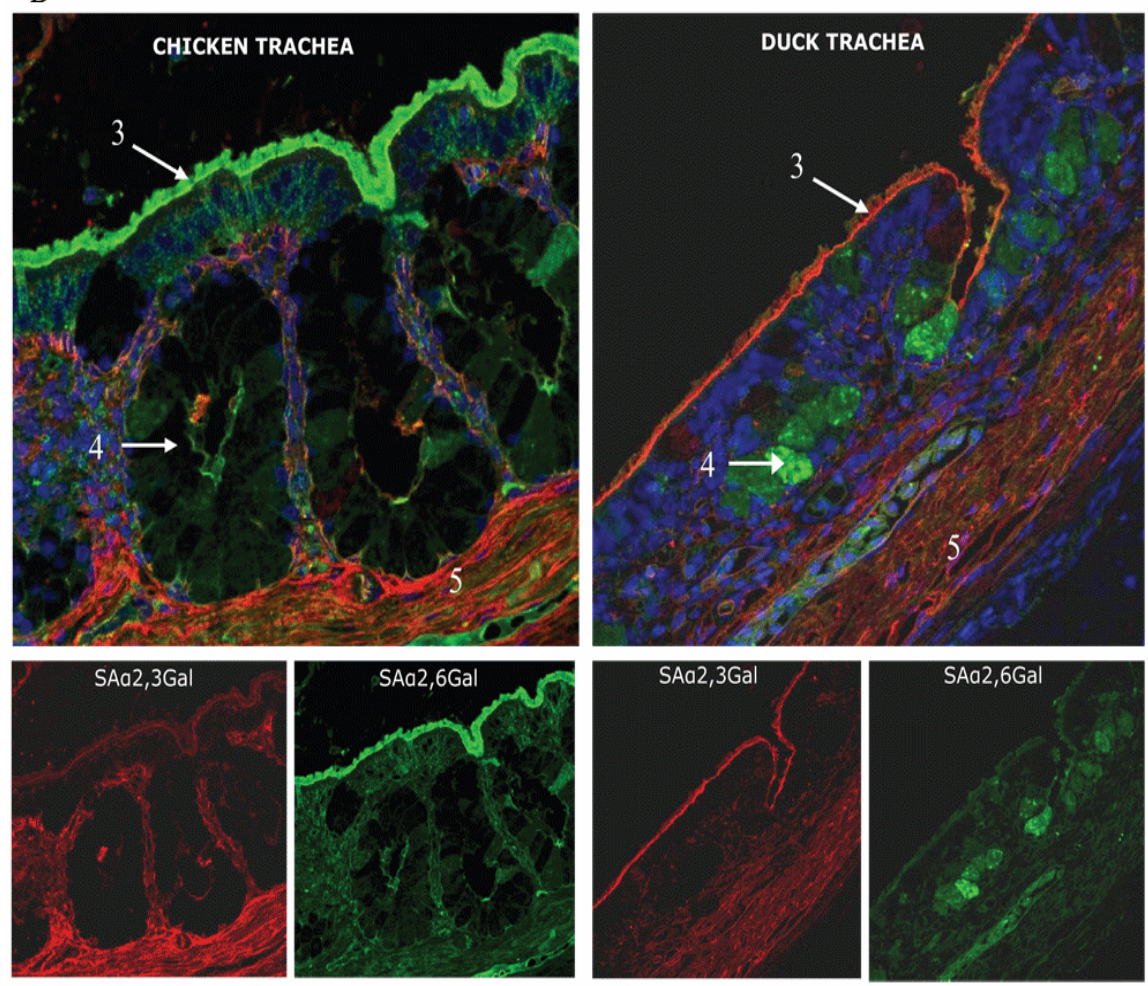

Figure 2. Differential expression of SA $2,6 \mathrm{Gal}$ and SA 2 2,3Gal receptors in tracheae and small intestine (SI) of chickens and ducks. (A) For both avian species, the epithelial lining of the SI shows predominant expression of SAa2,3Gal receptor (red), with little evidence of SA 2 2,6 Gal (green) expression. SA $2,6 \mathrm{Gal}$ receptors are more apparent in goblet cells of both bird types. Host receptor distribution is similar for both SI and large intestines (data not shown). (B) SA $\alpha 2,6 \mathrm{Gal}$ receptor is the more dominant type in chicken tracheal epithelium whereas $\mathrm{SA} \alpha 2,3 \mathrm{Gal}$ receptor is the main type in duck tracheal epithelium. 1. epithelial lining of the villus, 2. goblet cell, 3. epithelial cells (ciliated), 4. intra epithelial mucous gland, 5. submucosa. 
performed lectin staining on sialidase treated sections, which abrogated all staining confirming that the lectins used in the present study did not bind to non-sialic acid residues (data not shown). The difference in the predominant receptor across the tracheal epithelial lining in chicken and ducks could be an important contributing factor to influenza virus entry via the upper respiratory tract. In particular, such differences could impact on the susceptibility of each species to avian H5N1 influenza with its preferential tropism for infection of the respiratory tract rather than the intestines. The predominant receptor in the human upper airway is SA 2,6-Gal (Shinya et al, 2006; Yao et al, 2007). The high levels of expression of SA 2 2,6-Gal receptors in chicken tracheal epithelium suggests that this species may be more able to support the evolution of viruses with higher affinity for human SA 2 2,6-Gal receptors. This supports the suggestion that chickens may the source of emerging H9N2 viruses with a human-virus like receptor specificity (Gambaryan et al, 2002).

We further distinguished SAa2,3-Gal receptor subtypes, based on the third sugar residue, in chicken and duck trachea and intestines, with the use of MAA I (SA $\alpha 2,3-\mathrm{Ga}$ $\beta(1-4)$ GlcNAc specificity) and MAA II (SA $\alpha 2,3-G a l \beta(1-$ 3)GalNAc specificity). In chicken trachea, both SA 22,3 Gal receptor subtypes were detected in the sub-epithelial region (Figure $3 \mathrm{~A}$ ). However, along the chicken tracheal epithelium, SA $\alpha 2,3-G a l \quad \beta(1-4)$ Glc NAc receptor (MAA I lectin) was more dominant than SA $\alpha 2,3-\mathrm{Gal} \beta(1-3)$ Gal NAc receptor (MAA II lectin). In duck trachea, in contrast, minimal MAA I lectin binding was observed along the epithelium; only sub-epithelial mucous glands were MAA I positive. In duck trachea, SA $\alpha 2,3-G a l ~ \beta(1-3)$ Gal NAc receptor (MAA II lectin) was the main subtype detected, with distribution along the epithelial lining and in the mucosa (Figure 3A). In chicken large intestine, both SA $\alpha 2,3-G a l \beta 1-4$ Gal NAc receptor (MAA I lectin) and SA $\alpha 2,3-G a l$ 1-3 Glc NAc receptor (MAA II lectin) expression was observed. In duck large intestine, SA 2 2,3Gal $\beta 1-3$ Glc NAc receptor (MAA II lectin) was the main subtype detected, while the goblet cells were positive for SA $\alpha 2,3-G a l \quad \beta(1-4)$ Glc NAc receptor subtype (MAA I lectin) (Figure $3 \mathrm{~B}$ ). The binding pattern of MAA I and MAA II in the small intestines of both avian species was very similar to the large intestine (data not shown). In humans, MAA I shows more widespread binding throughout the upper and lower respiratory tract compared to MAA II (Nicholls et al, 2007). Avian influenza viruses isolated from chicken and ducks have been shown to preferentially bind to SA $\alpha 2,3-G a l \quad \beta(1-4)$ Glc NAc (recognized by MAA I) and SA $\alpha(2,3)$ Gal $\beta(1-3)$ Gal NAc (recognized by MAA II) respectively (Gambaryan et al, 2003). This reported virus tropism correlates with the observed receptor distribution in chicken and duck trachea and intestinal tissues. The presence of both SA $\alpha 2-3-G a 1$ receptor subtypes in chickens suggests that they may be susceptible to infection with wider range of avian influenza viruses with broader receptor specificity.

To relate the observed receptor distribution with the ability to bind viruses of avian or mammalian origin, we performed virus binding assays with avian $\mathrm{H} 2 \mathrm{~N} 3$ and swine H1N1 influenza viruses on tracheal and digestive tract sections. This showed the predicted preferential binding of the avian virus for SA 2 2,3-Gal receptor and the swine virus for SA $\alpha 2,6-\mathrm{Gal}$ receptors (Figure 4). The main SAa2,3-Gal receptor type in duck tracheal epithelium showed greater affinity for the avian $\mathrm{H} 2 \mathrm{~N} 3$ virus. In contrast, the dominant receptor type of SA 2,6 Gal in chicken trachea showed preferential binding of the swine H1N1 virus. The predominant expression of SA 2,3-Gal receptors type along the small and large intestinal epithelia of chickens and ducks showed preferential affinity for the avian $\mathrm{H} 2 \mathrm{~N} 3$ virus with no significant attachment of the swine virus. Virus-binding specificity was therefore consistent with host receptor type, as determined by lectin staining. The SA $22,6-\mathrm{Gal}$ receptor type expressed by the intestinal goblet cells did not appear seem to be functionally significant as no virus binding was observed with swine H1N1 virus (Figure 4).

The differences in receptor expression reported in this study suggest that they may be responsible, at least in part, for some of the differences between ducks and chickens in the pattern of disease following influenza infection. While the presence of a virus receptor is clearly not sufficient to confirm that cells or tissue support efficient virus replication or transmission, the widespread replication of influenza virus in multiple organs has been reported in both chickens (Swayne, 1997) and ducks (Londt et al, 2008) following infection with highly pathogenic viruses.

Genetic studies have revealed that previous pandemic influenza strains were partially or entirely derived from the viruses of avian origin (Webster et al, 1992 and Taubenberger et al, 1997, Horimoto and Kawawoka 2005). This study suggests that some chicken and duck tissues may facilitate entry of both human and avian viruses, with the ensuing danger of virus reassortment. However, further work is required to confirm that the tissues expressing both receptor types are able to support virus replication. The dominant presence of SA $\alpha(2,6) \mathrm{Gal}$ receptor along the chicken tracheal epithelium shows some similarities to the prevalence of the receptor in mammals such as human and pig. This suggests that chickens may be important intermediate hosts for the transmission of influenza to humans, in particular for influenza viruses such as H5N1, which show a respiratory tropism in birds. Whilst much attention has been placed on the role of pigs as "mixing vessels", the potential importance of chickens for the evolution of humanised influenza viruses should not be overlooked and, as such, warrants further study.

\section{CONCLUSIONS}

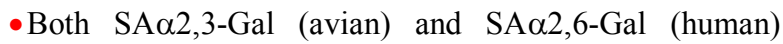
receptors are expressed in many tissues of chickens and ducks.

- SA 2 2,6-Gal receptor is the dominant receptor type in chicken tracheal epithelium, whereas in ducks the SA $22,3-$ Gal receptor is dominant.

- There is greater diversity of SA $2,3-\mathrm{Gal}$ receptor subtypes in chicken than duck. Chicken trachea and 
intestinal tissues showed positive binding with MAA I to MAA II whereas positive MAA II binding alone was noted across the epithelial lining in ducks. This suggests that chicken may be susceptible to infection with avian influenza viruses with broader receptor specificity.

- The host receptor distribution pattern in the chicken upper respiratory tract may be functionally significant for the evolution of viruses with a human like receptor specificity and thus for the transmission of influenza from birds and mammals.

\section{ACKNOWLEDGMENTS}

We are grateful to Dr Ian Brown and Dr Marek Slomka of Veterinary Laboratory Agency, Weybridge, UK for their help during the course of the work and kind supply of the influenza A viruses.

\section{COMPETING INTERESTS}

None declared.

\section{LIST OF ABBREVIATIONS}

DAPI: 4', 6-diamino-2- phenylindole, dihydrochloride DAB: diaminobenzidine

FITC: Fluorescein isothiocyanate

MAA: Maackia amurensis agglutinin

SA: Sialic acid

SNA: Sambucus nigra agglutinin
A
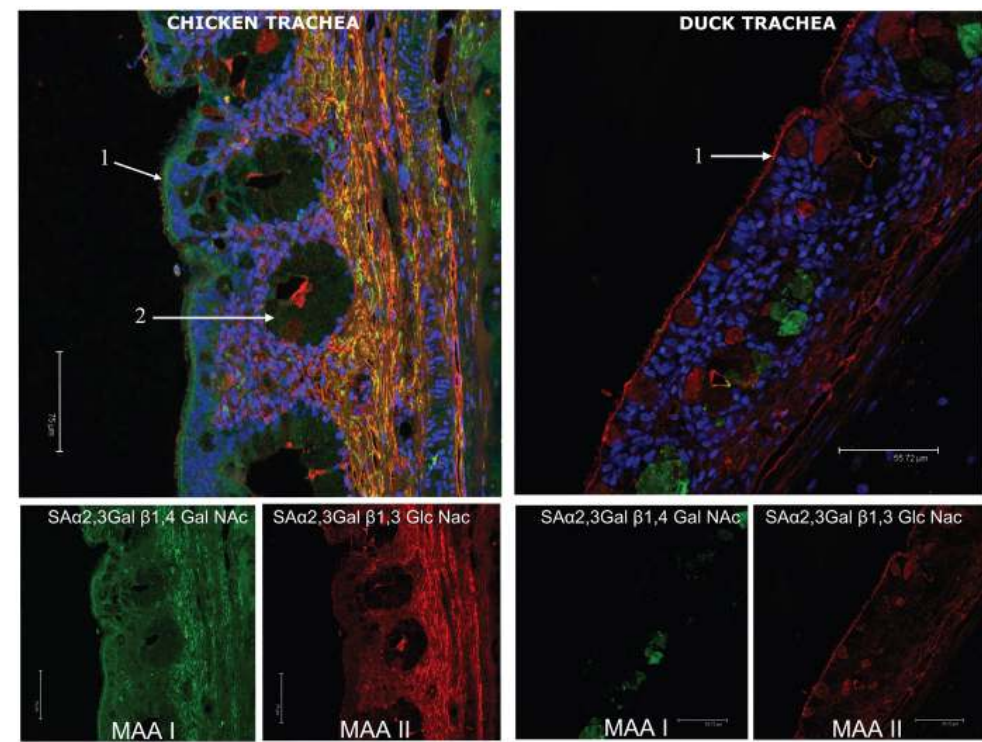

B
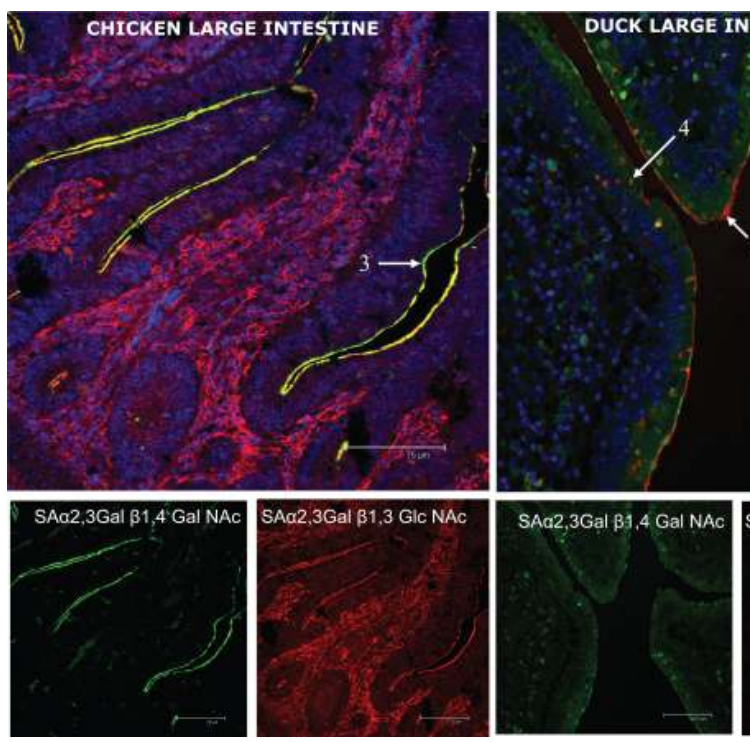

Figure 3. Differential expression of $\mathrm{SA} \alpha(2,3) \mathrm{Gal}$ receptor subtypes in chicken and duck tracheae and large intestines. MAA I (SA $\alpha(2,3)-G a l ~ \beta(1-4)$ Glc NAc detection; green) and MAA II (SA $\alpha(2,3)-G a l ~ \beta(1-3)$ Gal NAc detection; red) were used to distinguish $\mathrm{SA} \alpha(2,3)-\mathrm{Gal}$ receptor subtypes at the third sugar residue position. (A) Chicken trachea expresses $\mathrm{SA} \alpha(2,3)$-Gal $\beta(1-4)$ Glc NAc receptor (MAA I lectin) more strongly along the epithelial lining than SA $\alpha(2,3)$ Gal $\beta(1-3)$ Gal NAc (MAA II lectin). Both $\mathrm{SA} \alpha(2,3)$-Gal receptor subtypes are clearly present in the subepithelial region (see also MAA II lectin staining in Fig. 2B). In duck trachea, by contrast, minimal SA $\alpha(2,3)$-Gal $\beta(1-4)$ Glc NAc receptor subtype (MAA I lectin) is detected along the epithelium. Only the mucosal glands are MAA I positive. In duck trachea, SA $\alpha(2,3)-\mathrm{Gal}$ $\beta(1-3)$ Gal NAc receptor (MAA II lectin) is the main subtype with distribution along the epithelial lining and in the mucosa (see also MAA II lectin staining in Fig. 2B). (B) Chicken large intestine expresses similar levels of both $\mathrm{SA} \alpha(2,3)-\mathrm{Gal} \beta 1-4$ Gal NAc receptor (MAA I lectin) and $\mathrm{SA} \alpha(2,3)$ Gal $\beta(1-3)$ Gal NAc (MAA II lectin) along the epithelial lining. In duck large intestine, SA $\alpha(2,3)$-Gal $\beta(1-3)$ Gal NAc receptor (MAA II lectin) is the main subtype with distribution along the epithelial lining and in the mucosa. Similar observations were made in small intestines of chicken and duck (data not shown). 1. epithelial lining 2. intraepithelial mucous gland 3. epithelial lining of the villus 4 . goblet cell. 
Avian H2N3
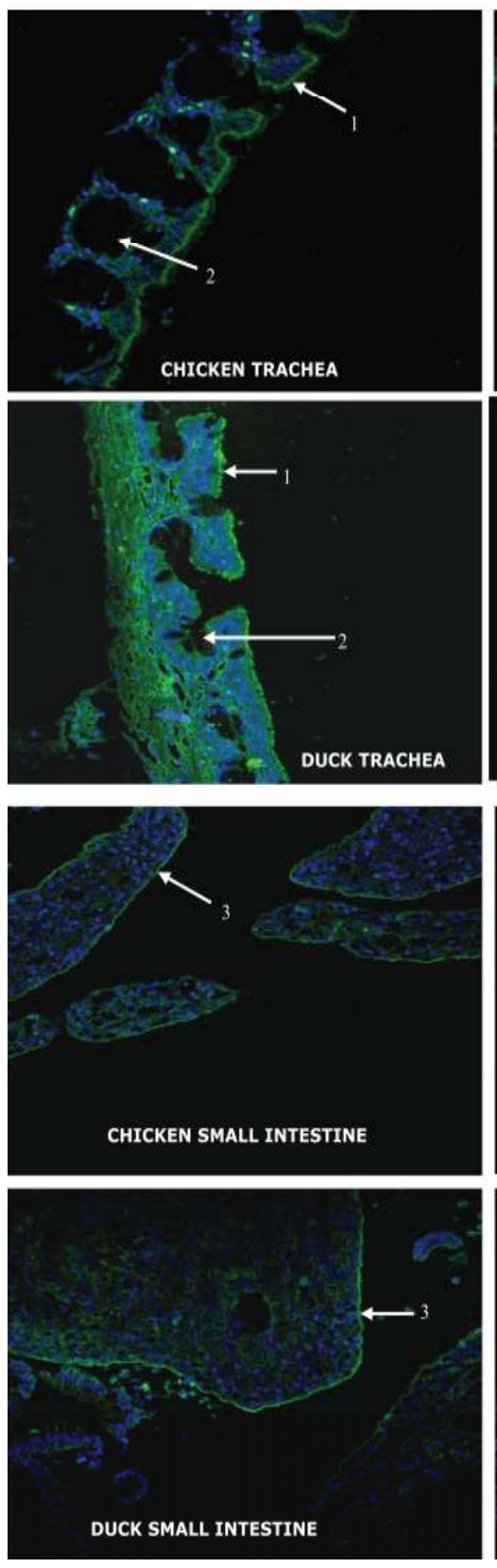

Swine H1N1
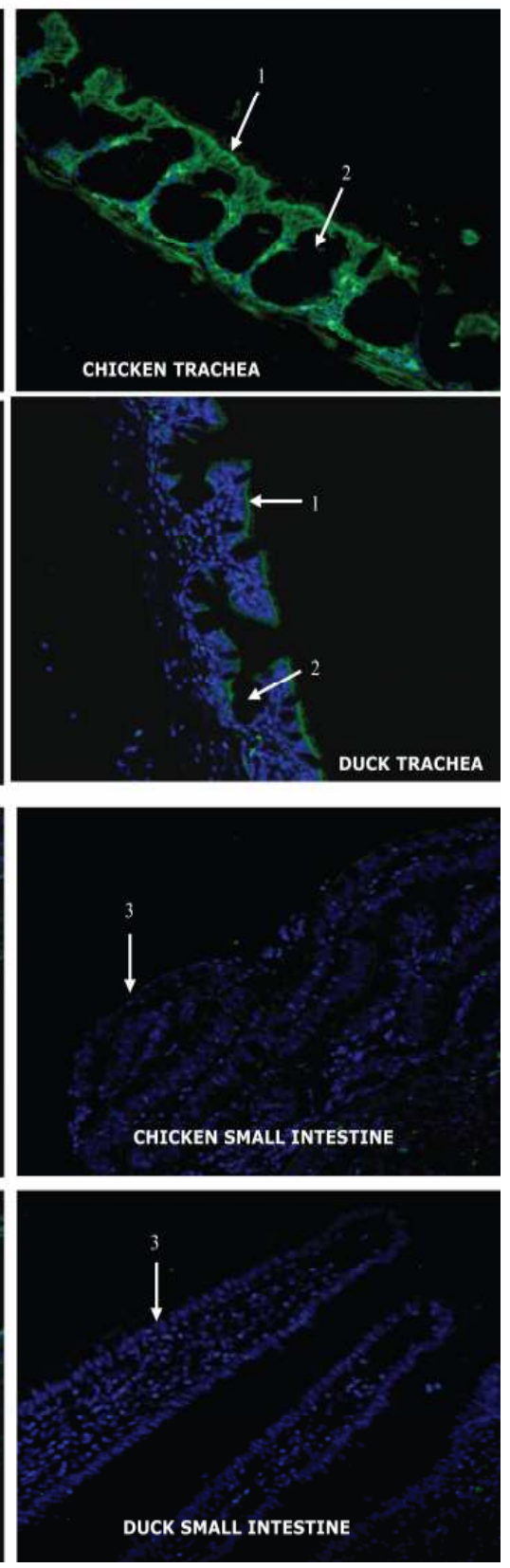

Figure 4. Virus binding assays with avian $\mathrm{H} 2 \mathrm{~N} 3$ and swine H1N1 influenza viruses on tracheal and intestinal tract sections reflect the observed receptor distribution. The main $\mathrm{SA} \alpha(2,3)-\mathrm{Gal}$ receptor type in duck tracheal epithelium shows greater affinity for the avian virus than the swine virus. By contrast, the dominant $\mathrm{SA} \alpha(2,6)$ Gal receptor type in chicken trachea shows preferential binding of the swine virus. The primary $\operatorname{SA} \alpha(2,3)$-Gal receptor type along the small intestine (SI) epithelia of both chickens and ducks is associated with preferential affinity for the avian virus, with no significant binding of swine virus. 1. epithelial lining, 2. intra-epithelial mucous gland, 3. epithelial lining of the villus.

\section{REFERENCES}

Claas EC, Osterhaus AD, Vanbeek R et al. 1998. Human influenza A H5N1 virus related to a highly pathogenic avian influenza virus. Lancet, 351, 472- 477.

Couceiro JN, Paulson JC and Baum LG. 1993. Influenza virus strains selectively recognize sialyloligosaccharides on human respiratory epithelium; the role of the host cell in selection of hemagglutinin receptor specificity. Virus Res, 29, 155-65.

Gagneux P, Cheriyan M, Hurtado-Ziola N et al. 2003. Humanspecific regulation of $\alpha 2-6$-linked sialic acids. J Biol Chem, 278, 48245-48250.

Gambaryan A, Webster R and Matrosovich M. 2002. Differences between influenza virus receptors on target cells of duck and chicken. Arch Virol, 147, 1197-1208.
Gambaryan AS, Tuzikov AB, Bovin NV et al. 2003. Differences between influenza virus receptors on target cells of duck and chicken and receptor specificity of the 1997 H5N1 chicken and human influenza viruses from Hong Kong. Avian Dis, 47, $1154-1160$.

Horimoto T and Kawaoka Y. 2005. Influenza: lessons from past pandemics, warnings from current incidents. Nat Rev Microbiol, 3, 591-600.

Ito T. 2000. Interspecies transmission and receptor recognition of influenza A viruses. Microbiol Immunol, 44, 423-430.

Ito T and Kawaoka Y. 2000. Host-range barrier of influenza A viruses. Veterinary Microbiology, 74, 71-75.

Ito T, Couceiro JN, Kelm S et al. 1998. Molecular basis for the generation in pigs of influenza A viruses with pandemic potential. J. Virol, 72, 7367-7373. 
Johnson, NPAS and Mueller J. 2002. Updating the accounts: global mortality of the 1918-20 'Spanish' influenza epidemic. Bull Hist Med. 76, 105-115.

Konami Y, Yamamoto K, Osawa T et al. 1994. Strong affinity of Maackia amurensis hemagglutinin (MAH) for sialic acidcontaining Ser/Thr-linked carbohydrate chains of N-termina octapeptides from human glycophorin A. FEBS Lett, 342, 334-338.

Löndt BZ, Nunez A, Banks J et al. 2008. Pathogenesis of highly pathogenic avian influenza A/turkey/Turkey/1/2005 H5N1 in Pekin ducks (Anas platyrhynchos) infected experimentally. Avian Pathol, 37, 619-27.

Matrosovich MN, Matrosovich TY, Gray T et al. 2004. Human and avian influenza viruses target different cell types in cultures of human airway epithelium. Proc Natl Acad Sci USA, 101, 4620-4624.

Nicholls JM, Bourne AJ, Chen H et al. 2007. Sialic acid receptor detection in the human respiratory tract: evidence for widespread distribution of potential binding sites for human and avian influenza viruses. Respiratory Research, 8, 73.

Perdue ML and Swayne DE. 2005. Public health risk from avian influenza viruses. Avian Dis, 49, 317-27.

Shibuya N, Goldstein IJ, Broekaert WF, Simba-Lubaki MN et al. 1987. The elderberry (Sambucus nigra L) bark lectin recognizes the Neu5Ac (alpha 2-6) Gal/Gal Nac sequence. J Biol Chem, 262, 1596-1601.

Shinya K, Ebina M, Yamada S, et al. 2006. Influenza virus receptors in the human airway; avian and human flu viruses seem to target different regions of a patient's respiratory tract. Nature, 440, 435-436.

Subbarao K, Klimov A, Katz J et al.1998. Characterization of an avian influenza A (H5N1) virus isolated from a child with a fatal respiratory illness. Science, 279, 393-396.

Swayne, DE. 1997. Pathobiology of H5N2 Mexican avian influenza virus infection in chickens. Vet Pathol, 34, 557-567.

Taubenberger JK, Reid AH, Krafft AE et al. 1997. Initial genetic characterization of the 1918 "Spanish" influenza virus. Science, $275,1793-1796$

Taubenberger JK, Reid AH, Lourens, RM et al. 2005. Characterization of the 1918 influenza virus polymerase genes. Nature, 437, 889-893.

Tumpey TM, Basler CF, Aguilar PV et al. 2005. Characterization of the reconstructed 1918 Spanish influenza pandemic virus. Science, 310, 77-80.

Wan H and Perez DR. 2006. Quail carry sialic acid receptors compatible with binding of avian and human influenza viruses. Virology, 346, 278-286.

Webster RG, Bean WJ, Gorman OT et al.1992. Evolution and ecology of influenza A viruses. Microbiol Rev, 56, 152-179.

Webster RG, Shortridge KF and Kawaoka Y. 1997. Influenza: interspecies transmission and emergence of new pandemics. FEMS Immunol Med Microbiol, 18, 275-279.

Yao L, Korteweg C, Hsueh W et al. 2008. Avian influenza receptor expression in $\mathrm{H} 5 \mathrm{~N} 1$-infected and noninfected human tissues. FASEB J, 22, 733-740. 